\title{
Metodología para medir la pobreza en Moldavia (Rumania) ${ }^{1}$
}

\author{
Daniel Tudora ${ }^{2}$
}

\begin{abstract}
RESUMEN
La pobreza comunitaria, analizada como la suma de varios tipos de privación social y territorial, es la expresión geográfica de distintos procesos y fenómenos, y comúnmente es objeto de estudios sociológicos y económicos. En el presente estudio se realizó la condensación estadística de una serie de variables primarias, generándose un indicador sintético, superior desde el punto de vista informativo, que se denomina índice del desarrollo comunitario. La validación estadística de los resultados está acompañada por una validación espacial, identificando el comportamiento de estructuras sociales legítimas en áreas rurales, destacando que la obtención de resultados válidos en la implementación de estrategias de desarrollo territorial dependen más de la consistencia de los métodos científicos utilizados que de interpretaciones estadísticas certeras.
\end{abstract}

Palabras clave: Pobreza comunitaria, disparidades regionales, métodos estadísticos.

\begin{abstract}
Community poverty, analyzed as the sum of several types of social and territorial deprivation, is the geographical expression of various processes and phenomena, commonly the object of sociological and economic studies. In this paper we performed a statistical compaction of a series of synthetic indicators, generating an indicator called the index of community development. The statistical validation of these results is accompanied by a spatial validation, which identifies the legitimate social structures in rural areas, emphasizing that obtaining valid results in the implementation of territorial development strategies depends more on the consistency of the scientific methods used to interpret statistical analysis.
\end{abstract}

Key words: Community poverty, regional disparities, statistical methods.

1 Artículo recibido el 2 de diciembre de 2011, aceptado el 24 de julio de 2012 y corregido el 28 de junio de 2013.

\footnotetext{
2 Departamento de Geografía, Universidad Alexandru Ioan Cuza (Rumania).

E-mail: tudoradaniel@yahoo.com
} 
La geografía social como disciplina que forma parte de la geografía humana intenta, en cuanto al problema de la pobreza, ofrecer las explicaciones de una cierta repartición espacial del subdesarrollo, y favorece el análisis horizontal del fenómeno, demostrando por medio de métodos propios que la pobreza es también un fenómeno espacial que respeta ciertas leyes de distribución, ordenación y coordinación en el territorio, que es creada por la geografía y que a su turno, crea geografía.

Las formas más recientes del análisis de la pobreza ponen la atención en el descubrimiento de la insuficiencia del fenómeno y busca métodos complexos de enfoque. La delimitación de las clases de pobreza en función de los ingresos comienza a ser considerada como un trámite reduccionista porque ignora la presencia de otras necesidades, movilidades vitales verticales del individuo: el sentimiento de seguridad, la libertad de la expresión, el acceso a la educación, al sistema sanitario, al medio natural sanogeno, etc., factores que el economista indio Amartya Sen Ilama comodidades. Ulteriormente, se desarrolló toda una literatura de métodos que completan diversos aspectos del estudio de la pobreza o que intentan crear indicadores sintéticos con cobertura más amplia del fenómeno: la pobreza de la infancia (Minujin, 2006), la pobreza femenina (Daly, 1989), la pobreza relativa (Smeeding \& O'Higgins, 1990).

Desde este punto de vista epistemológico, la pobreza se transforma en un tema multidimensional y los índices correspondientes tienen una estructura heterogénea acercando cada vez más el concepto de nivel de vida, sin eludirlo, al concepto de nivel de existencia: el índice multidimensional de pobreza, índices ajustados al nivel de desigualdad o el índice de las necesidades básicas insatisfechas (el último muy utilizado en América Latina).

Las preocupaciones para la dimensión espacial de la pobreza son también las más recientes, el análisis geográfico del fenómeno siendo muchas veces integrado a los estudios de tipo económico o sociológico, derivados de la necesidad de explicar la aparición de algunas formas localizadas de la pobreza, a veces por la iniciativa de las estructuras afilia- das a la ONU en su intento de trazar los perfiles de la pobreza (Lok-Desallien, 2004). Un ejemplo lo representa la pobreza rural, que se propone explicar las particularidades del más repartido tipo de pobreza, los estudios que se refieren a los procesos, a las causas y a los efectos de la frecuencia con la que la pobreza se asocia al medio rural siendo presentes en todas las escuelas especializadas del mundo (Jazairy et al., 1995).

El desarrollo de los métodos de computación de los datos espaciales permitió a la geografía moderna desarrollar sur propios métodos de análisis de la repartición de la pobreza, el mapa transformándose para la geografía de una simple solución de visualización de la información estadística en un método de investigación, la disciplina llamada análisis espacial. Centrada en el estudio de las distancias, de la contigüidad, de las discontinuidades y de la accesibilidad, el análisis espacial propone varios métodos de estudio de la repartición de la pobreza de los cuales los más conocidos están circunscritos por los modelos centro-periferia, de la difusión espacial de la innovación y de los de la proximidad, cada uno de ellos proponiendo varias técnicas de análisis: la autocorrelación espacial, la regresión geográfica ponderada, la interacción espacial (Pérez, 2005; Anselin, 2005).

Las ciencias del espacio benefician de la ventaja de integrar la información social y económica en arquitecturas con disposición horizontal, asimilando los individuos en grupos humanos estrictamente localizados pero descritas sutilmente por medio de unos indicativos de tipo topológico: relees, masa y volúmenes descritos por puntos, redes de informaciones y relaciones de emisión y recepción descritas por líneas, superficies, capitales y patrimonios descritos por polígonos.

En el interior de estas referencias concretas, el geógrafo dispone de las competencias necesarias para discriminar algunas desigualdades que la sociedad secreta por repetitividad / autorregeneración, flexibilidad/ rigidez, simplicidad/ complejidad, mezclando métodos y modelos de la economía, sociología, historia, que integra sinérgicamente en un volumen de información cartografiable Ilamado capital espacial. 
La ausencia/carencia de capital espacial produce una especie geográfica del subdesarrollo llamada pobreza comunitaria.

\section{Metodología del cálculo del índice de subdesarrollo}

Las hipótesis de este tipo de estudio resultan de la dificultad de circunscribir al mismo tiempo varias categorías de indicadores que analizan los problemas del subdesarrollo comunitario - económico, social o geográfico.

Las clasificaciones a base de análisis multicriterios se ponen aún más difíciles en el contexto de la presencia de varias relaciones dependientes de su dimensión territorial. En general, las principales dificultades encontradas son:

- la utilización alternativa de los indicadores sintéticos que llevan a convenios paramatemáticos, discutibles, de modo que variables con origen explicativo diverso se utilizan con la misma importancia en el resultado final, lo que puede llevar a conclusiones arbitrarias (Jean Gadrey, 2002).

- la ponderación sucesiva de los subindicadores del subdesarrollo, utilizan métodos estadísticos subjetivos, de tipo marcas, que eluden la energía real que se establece entre variables.

- la aplicación de las análisis multivariadas no tienen en cuenta las particularidades esenciales de las series de datos territoriales que retienen gran parte de la información inexplicada, invisible a su simple correlación o a la simple comparación con su perfil medio.

Clásicamente, la economía y a veces la sociología estudian la pobreza por medio de la información sobre los ingresos y el consumo de la población, y a pesar de varias discusiones relacionadas con la manera de definir la pobreza, la mayoría de las fuentes ofrecen como punto de partida en la investigación del fenómeno los datos sobre las fuentes de ingreso de la población (Nolan \& Whelan, 2007; Atkinson, 2002).

Más o menos atentas al carácter exhaustivo de las investigaciones que colectan datos sobre ingresos, tanto la economía como la sociología intentaron delimitar niveles estadísticos que declinen la población en función de las posibilidades financieras o hasta sociales. Dos de estas son las más aceptadas en la literatura de especialidad: el nivel de pobreza absoluta, establecido en función de una cesta mínima de bienes necesarios al funcionamiento normal del individuo en la sociedad (Townsend \& Gordon, 1991, Jansson, 2000) y el nivel de pobreza relativa (Sen, 1983), que considera que la pobreza debe ser analizada desde la perspectiva de la sociedad en la que vive el individuo, utilizando distancias estadísticas en relación a ciertos valores centrales, de los cuales el más utilizado es la mediana de los ingresos (Townsend, 1979; Mack, 1985).

La primera parte del artíuculo se concentra en la delimitación de los componentes del medio económico y social que a priori presentan interés para la delimitación de las zonas afectadas por la pobreza comunitaria. Su condensación estadística debería eliminar la redundancia entre las variables primarias y obtener un indicador sintético, superior desde punto de vista informativo, que se Ilamará índice del desarrollo comunitario. Las variables primarias utilizadas en el estudio están agrupadas en seis capítulos de análisis, generándose para cada capítulo un indicador parcial:

1. Índice de accesibilidad en relación a los servicios educacionales

2. Índice de accesibilidad en relación a los servicios sanitarios

3. Índice de accesibilidad en relación a los servicios financieros bancarios

4. Índice de accesibilidad en relación al mercado laboral

5. Índice de rentabilidad de las explotaciones agrícolas en conformidad con la accesibilidad al mercado de los productos primarios

6. Índice de la calidad de vida

Cada uno de estos indicadores parciales se centra en la idea de la accesibilidad potencial, concepto que supone la relación entre dos vectores complementarios: uno de emisividad y uno de atracción. La manera en que los dos vectores se completan recíprocamente en el espacio tiene en cuenta las masas humanas puestas en interacción, su calidad y 
por supuesto la distancia que separa dichas masas.

La accesibilidad será valorada a lo largo del trabajo en la calidad de una distanciatiempo, calculada al nivel de las vías de comunicación por carretera, considerando que el desplazamiento se realiza con medios auto, en condiciones ideales de tráfico.

La dicha información fue tratada al nivel de localidad, 2.944 asentamientos humanos, que totalizan las ocho provincias del este de Rumania que forman la región histórica Ilamada Moldova.

\section{Tipología del subdesarrollo de Moldova mediante el análisis integrado de los índices parciales}

El capítulo final demuestra varios métodos de análisis multivariado para crear un indicador final del desarrollo comunitario que beneficie de una parte de la ventaja de obtener una síntesis de todos los segmentos del desarrollo presentados en los capítulos anteriores, y de otra parte tener la calidad de extraer ciertas leyes de la manifestación de la pobreza comunitaria en el espacio. Desde este último punto de vista, se desea que el indicador tenga valores con referencias claras y que pueda aplicarse también en el caso de otras regiones del mundo.

Después de testar varios métodos entre otros, el análisis cluster y el método de la suma de los rangos, el autor prefiere el análisis factorial porque este permitió subrayar el hecho de que los indicadores parciales no participan con la misma ponderación a la formación de un perfil del desarrollo comunitario de las localidades estudiadas.

Al contrario, el efecto de colinealidad entre los indicadores parciales certifica el hecho de que la participación del indicador de accesibilidad a los servicios sanitarios está reducido en comparación con los seis indicadores parciales.

De conformidad con el análisis por componentes principales, se llegó a la conclusión de que se puede reducir el número de seis variables directas expuestas anteriormente a 4 factores que aseguran el $90 \%$ de la explicación de toda la dispersión. Después de tomar de la matriz de conexión los valores propios de cada factor, se notaron dos aspectos:

\section{¿Cuál es la correlación entre las variables} directas?

Por ejemplo, al analizar la correlación entre las seis variables, se nota la presencia de dos tipos de correlaciones entre las variables iniciales incluidas en la investigación :

- Se establecen correlaciones directas entre el índice de los servicios educacionales, el índice técnico-edilicio de las propiedades, el índice de mercado de la fuerza de trabajo y el índice financiero-bancario, la más estrecha correlación es entre el índice técnico-edilicio y el índice financierobancario.

- Se puede notar una correlación inversa entre el factor de los servicios sanitarios y el factor del beneficio de las explotaciones agrícolas, la disminución del primero implicando también la probabilidad de una erosión de la estructura por grupos de edades, lo que influye positivamente el aumento del segundo índice.

¿Cuál es la importancia de cada variable para determinar los cuatro componentes principales del análisis?

Para poner en evidencia las relaciones de inigualdad que se establecen entre las variables latentes del índice de desarrollo comunitario, se utilizará al principio un análisis estadístico exploratorio que tiene como objetivo establecer la importancia de cada factor en función del promedio de las correlaciones entre las variables.

¿Cuál es el peso de cada variable en la determinación de los cuatro componentes principales del análisis?

Para poner de relieve las relaciones de desigualdad que se establecen entre las variables latentes del índice de desarrollo de la comunidad, se utilizará inicialmente un estudio estadístico de sondeo que tiene el objetivo de determinar la importancia de cada 
factor, basado en correlaciones medias entre las variables.

El método se puede expresar sintéticamente según una matriz en la que están representados los correlogramas de los seis índices parciales llevados dos a dos.

Los enlaces que se muestran más fuertes están evidenciados entre el factor de la accesibilidad a los servicios bancario-financieros y el factor de la calidad de servicios públicos del hábitat, aspecto que pone de relieve el papel fundamental que tiene la variable número de personas que se fueron al extranjero a trabajar en la realización de los presupuestos de las comunidades rurales.

Los procesos de estadística matemática no pueden identificar estas relaciones que se esconden más allá de la interfaz cuantitativa de las variables latentes que están ofrecidos para procesamiento; las deficiencias observadas fueron causadas por un defecto esencial en la recogida de las bases de datos territoriales imputación estadística.

Las respectivas correlaciones pueden ser traducidas a la siguiente ecuación, la escasa importancia de los factores salud y agroalimentario siendo deducible además del carácter particular de estas dos variables en el marco bivariado de los dos primeros componentes principales del análisis factorial.

$$
\begin{gathered}
I_{d c}=0.220 \cdot I_{e d}+0.272 \cdot I_{f b}+0.237 \cdot I_{t e}+0.108 \\
\cdot I_{\text {pea }}+0.190 \cdot I_{p f m}+0.045 \cdot I_{s s},
\end{gathered}
$$

donde:

$I_{d c}-E l$ índice del Desarrollo de la Comunidad, $I_{\text {ed }}$ - El índice de los servicios educativos, $\mathrm{I}_{\mathrm{fb}}$ - El índice de la banca, $\mathrm{I}_{\mathrm{ts}}$ - El índice de la infraestructura técnica, $I_{\text {pea }}$ - El índice de ingresos de la Granja, $I_{\mathrm{pfm}}$ - El índice del mercado de trabajo, $\mathrm{I}_{\mathrm{ss}}$ - El índice de servicios de salud.

Por la continuación del método de análisis se dará lugar a la eliminación de dos variables insignificantes en la explicación de la varianza total, con la debida recuperación de las variables explicativas principales.
La deficiencia de tal proceso es reclamado por la naturaleza estocástica en función de la cual se separan las variables con un alto valor explicativo de las con valor explicativo escaso, perdiendo así dos de las variables latentes.

Además, la realidad geográfica muestra que, a diferencia de las estadísticas geométricas, las series de datos regionales se destacan por las relaciones de tipo inferencial en las cuales las variables se analizan en una constante evolución sinérgica.

Si esto es validado por la información estadística geográfica específica, resulta que dentro del análisis territorial, los factores que, al parecer son los menos explicativos, gracias a mostrarse con más independencia hacia otras variables, se mostrarán de mayor elevación en el valor final del índice de desarrollo; estas relaciones con la Comunidad se pueden derivar matemáticamente de la matriz de conexión de las variables y los cuatro principales factores destacados por el modelo del análisis en componentes principales.

La ecuación del índice de desarrollo de la comunidad:

$$
\begin{aligned}
I_{d c}= & 13.57 \cdot I_{e d}+13.56 \cdot I_{f b}+13.57 \cdot I_{t e} \\
& +16.15 \cdot I_{p e a}+16.47 \cdot I_{p f m}+16.67 \cdot I_{S S}
\end{aligned}
$$

La validación estadística de los resultados está acompañada por una validación espacial, el indicador final del desarrollo comunitario siendo testado en un espacio de referencia (una zona rural de la provincia de Botosani), identificando las principales características de los espacios afectados por la pobreza comunitaria:

- Se desarrollan más allá del campo gravitatorio creado por la ciudad;

- La distancia a la ciudad está determinada por el tamaño y las funciones del centro urbano, las ciudades pequeñas permiten límites más flexibles, mientras que las grandes ciudades obligan a la contracción de estos límites hacia el centro del territorio;

- Se eviten las vías de comunicación de mayor rango (carreteras europeas, carretera nacional); 
- Se pueden invadir los espacios intermedios, si estos son jóvenes, no evolucionado o intermitente;

- Ellos se diferencian entre sí en términos de desarrollo de la comunidad por tamaño, los índices de desarrollo más bajos de la comunidad aparecen dentro de los mayores espacios rurales repulsivas.

La parte final del trabajo presenta las conclusiones bajo la forma de unas leyes de la manifestación en el espacio del desarrollo comunitario

L1 - El desarrollo comunitario de las localidades depende de la accesibilidad a los servicios de rango inferior y medio, las localidades de rango superior introducen diferencias intraclase, en el nivel superior de la jerarquía.

L2 - El desarrollo comunitario de los asentamientos rurales en función de la proximidad a la ciudad.

L3 - El desarrollo comunitario responde al efecto de masa.

L4 - El desarrollo comunitario es una función - tiempo que describe la probabilidad de interacción entre los asentamientos rurales y el sistema mayor de las vías de comunicación de una región/Estado.

L5 - El desarrollo comunitario es la suma transescalar de unas accesibilidades específicas.

L6 - El desarrollo comunitario es el efecto cúmulo de las percepciones y de los sentimientos negativos/positivos de la población sobre cierta zona, el resultado de estos esquemas mentales siendo "el marcaje territorial"

\section{Referencias bibliográficas}

CHAVES, A.; SANCHEZ, S.; SCHOR, S. \& TESLIUC, E. Financial markets, Credit Constraints and Investment in Rural Romania. Washington: World Bank Technical Papers, 2001.

CHIRAN, A. Piaţa produselor agricole şi agroalimentare - abordare teoretic ă şi practicăa, Bucureşti: Ed. Ceres, 2004.

COLEMAN, J. Social capital in the creation the human capital. American Journal of Sociology, 1988, № 94, p. 95-121.
DUPUY, G. L'urbanisme des reseaux, theories et methodes. Paris: Ed. Armand CoIlin, 1991.

ESPING-ANDERSEN, G. The Three Worlds of Welafare Capitalism. Ney Jersey: Princeton University Press, 1990.

FLORES, M. y RELLO, F. Capital social: virtudes y limitaciones. Santiago de Chile: CEPAL, 2003.

GADREY, J. \& JANY-CATRICE, F. Les indicateurs de richesse et de développement. Un bilan international en vue d'une initiative française. Lille: Rapport de recherche pour la DARES, 2003.

GADREY, J. De la croissance au developpment: quels indicatuers alternatifs? Lille: Universite Lillle, 2002.

GOSCHIN, Z. \& PARLOG, C. lerarhizarea multicriterială a regiunilor din România folosind metoda distanţelor relative. Bucureşti: Ed. Economică, 2004.

HERBERT, S. Models of Bounded Rationality. Cambridge, MIT Press, 1997.

KATZMAN, R. y FILGUEIRA, C. Vulnerabilidad, Activos y Exclusion Social en Argentina y Uruguay. Santiago de Chile: OIT-Fundacion Ford, 1999.

DELALANDE, L. \& PAQUETTE, C. El impacto de las microfinanzas en la reduccion de la vulnerabilidad. Trace, 2007, № 52, p. 63-77.

NELEA, N.M. Caracterizarea calităţii vieţii în România prin prisma statisticii multidimensionale. Bucureşti: Ed. ASE, 2004.

PAUGAM, S. La disqualification sociale. Essai sur la nouvelle pauvreté. Paris: Presses Universitaires de France, 1997.

PELLEN, M. Penser les espaces intermediaires. Lyon: Calenda, 2009.

PUMAIN, D. \& SAINT-JULIEN, T. Les interactions spatiales. Paris: Ed. Armand Collin, 2001. 
PUTNAM, R. The prosperous community: social capital and public life, American prospect, 1993, $\mathrm{N}^{\circ} 3$, p. 35-42.

REYNAUD, A. Société, espace et justice. Inégalités régionales et justice socio-spatiale. Paris : PUF, 1981.
SEN, A. Commodities and Capabilities. London: Cambridge University Press, 1999.

VOINEAGU, V. \& FURTUNA, F. Analiza factorială a fenomenelor social-economice în profil regional. Bucureşti: Ed. Aramis, 2002. 
